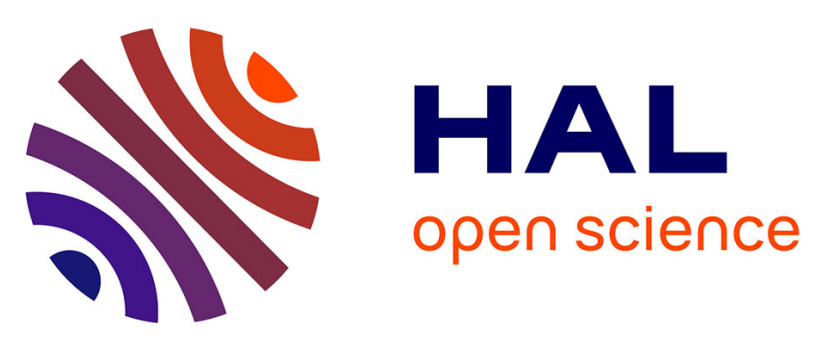

\title{
Les signes d'écriture tracés au poinçon (kakuhitsu ) à l'époque de Nara. Présentation de récentes découvertes faites par l'équipe du professeur Kobayashi Yoshinori , autour de manuscrits du VIIIe siècle conservés au Shōsōin de Nara
}

\author{
Frédéric Girard
}

\section{To cite this version:}

Frédéric Girard. Les signes d'écriture tracés au poinçon (kakuhitsu ) à l'époque de Nara. Présentation de récentes découvertes faites par l'équipe du professeur Kobayashi Yoshinori , autour de manuscrits du VIIIe siècle conservés au Shōsōin de Nara. Bulletin de l'Ecole française d'Extrême-Orient, 2005, 92 (1), pp.571-577. 10.3406/befeo.2005.5999 . hal-02536679

\author{
HAL Id: hal-02536679 \\ https://hal.science/hal-02536679
}

Submitted on 8 Apr 2020

HAL is a multi-disciplinary open access archive for the deposit and dissemination of scientific research documents, whether they are published or not. The documents may come from teaching and research institutions in France or abroad, or from public or private research centers.
L'archive ouverte pluridisciplinaire HAL, est destinée au dépôt et à la diffusion de documents scientifiques de niveau recherche, publiés ou non, émanant des établissements d'enseignement et de recherche français ou étrangers, des laboratoires publics ou privés. 


\section{per

Les signes d'écriture tracés au poinçon (kakuhitsu 角筆) à l'époque de Nara. Présentation de récentes découvertes faites par l'équipe du professeur Kobayashi Yoshinori 小林芳規, autour de manuscrits du VIIle siècle conservés au Shōsōin de Nara Frédéric Girard

\section{Citer ce document / Cite this document :}

Girard Frédéric. Les signes d'écriture tracés au poinçon (kakuhitsu 角筆) à l'époque de Nara. Présentation de récentes découvertes faites par l'équipe du professeur Kobayashi Yoshinori 小林芳規, autour de manuscrits du VIIle siècle conservés au Shōsōin de Nara. In: Bulletin de l'Ecole française d'Extrême-Orient. Tome 92, 2005. pp. 571-577;

doi : https://doi.org/10.3406/befeo.2005.5999

https://www.persee.fr/doc/befeo_0336-1519_2005_num_92_1_5999

Fichier pdf généré le 08/02/2019 


\title{
Les signes d'écriture tracés au poinçon (kakuhitsu 角筆) à l'époque de Nara. \\ Présentation de récentes découvertes faites par l'équipe du professeur Kobayashi Yoshinori 小林芳規, autour de manuscrits du VIII ${ }^{\mathrm{e}}$ siècle conservés au Shōsōin de Nara
}

\author{
Frédéric GIRARD
}

Les poinçons sont des instruments d'écriture faits d'ivoire, de bois, de bambou ou de corne de daim dont on aiguise une extrémité afin de s'en servir comme d'un 《 pinceau dur », d'un " pinceau cornu ou anguleux » (kakuhitsu角筆), à l'aide duquel on trace en creux, sur une feuille de papier ou sur un papyrus, des signes, des dessins, des lignes mélodiques de partitions musicales ou des caractères d'écriture. Un des intérêts de l'utilisation de cet instrument est que les signes ainsi tracés sont lisibles à travers la feuille de papier déployée face à une source lumineuse diurne ou artificielle, sans que, pour autant, la calligraphie des caractères chinois ou japonais soit endommagée. Ce type de notation de partitions musicales ou de caractères d'écriture sera graduellement remplacé par la suite par la notation de signes faite au pinceau à l'encre, blanche ou colorée (principalement, tout d'abord, rouge vermillon), sur des textes en chinois, de provenance principalement bouddhique, lorsqu'une importance moindre sera accordée à ces textes sacrés. Les textes conservés au Shōsōin 正倉院, qui est le trésor attitré abritant des objets précieux ayant appartenu aux empereurs et impératrices de l'époque de Nara (714-784), renferment le Canon bouddhique que l'impératrice Kōmyō 光明皇后 (701-760) avait demandé de faire copier, à titre d'œuvre pieuse et méritoire, au milieu du viII ${ }^{\mathrm{e}}$ siècle. Ce travail de copie, sacré par excellence - en cette période d'imitation de la Loi ( $z \bar{o} b \bar{o}$ 像法), la copie de sütra était l'une des œuvres pies permettant d'accéder en une vie prochaine à un paradis, à une Terre pure d'où l'on aurait accès au Nirvāṇa -, ne supportait bien entendu pas de subir les dommages d'une sur-écriture au pinceau : tracer des signes d'écriture sur les caractères chinois de sütra pouvait être ressenti comme une sorte de profanation de ces textes, altérant les caractères. On comprend donc aisément que, si l'on avait à y faire figurer des signes de lecture, on ait préféré utiliser un système de notation invisible à l'œil nu plutôt qu'à l'encre.

Or, on a trouvé un tel système de notation sur des copies du Sütra de l'Ornementation fleurie dans sa version en quatre-vingts volumes (Huayanjing 華嚴經), ainsi que sur son commentaire composé par Huiyuan 慧苑 (673-743), le Collationnement du Commentaire abrégé [de Fazang] sur le Sütra de l'Ornementation fleurie (Huayanjing lueshu kandingji 華嚴經略疏刊定記). Ces deux ouvrages avaient fait l'objet de lectures commentées, en rapport étroit avec l'érection du Buddha géant du Tōdaiji, 
par le moine Shinjō [cor. Simsang] 審祥 (?-?) ${ }^{1}$, qui était allé étudier dans le royaume coréen de Silla. Shinjō avait entrepris ses lectures à partir de 740, à l'instigation de la cour impériale, et celles-ci ont continué jusqu'en 789 ; le commentaire de Huiyuan a été lu, lui, de 743 à 788 , et c'est sur les volumes V et IX qu'on a retrouvé des signes de lecture et d'inversion du type du Silla. C'est naturellement qu'il est venu à l'esprit des savants de mettre en rapport ces signes de lecture portés sur les textes de la littérature de l'Ornementation fleurie, lus et commentés avec assiduité et constance durant plusieurs décennies tout d'abord par un religieux japonais s'étant rendu au Silla, avec ceux qu'on pouvait trouver dans des textes bouddhiques du Silla ou provenant de ce royaume coréen. Les enquêtes ont également porté sur d'autres catégories de textes qui attestent eux aussi un tel emploi du poinçon, même si c'est cette activité de lecture au Tōdaiji qui semble en avoir été initialement le centre.

C'est au sein de tels textes à caractère canonique que récemment, le professeur Kobayashi Yoshinori 小林芳則, éminent philologue, spécialiste de l'histoire de l'écriture japonaise à l'époque ancienne, a déchiffré des signes de lecture des caractères chinois, pour la transcription du japonais et du coréen - signes de lecture des caractères, des particules et des mots outils, signes d'inversion, signes de finales, etc. - , connus par la suite sous le nom de wokototen $ᄏ コ$ ト點. Le caractère tardif de cette découverte s'explique par le fait que ces textes, conservés au Département des affaires impériales (Gūnaichō 宮内庁), ne sont pas accessibles au public, pas même au public savant : le professeur Kobayashi déclare que lui-même ne peut emprunter des rouleaux, des volumes, ou parfois seulement des feuillets, de ces textes sacrés qu'au compte-goutte, ce qui ralentit et gêne son travail de dépouillement; le déchiffrement de ces signes demande une expérience considérable des textes que les savants ou conservateurs de musée qui l'ont précédé n'avaient réussi à mettre en évidence. C'est l'opiniâtreté d'un savant d'exception - il travaille sur ce type de documents depuis une quarantaine d'années et en a présenté une synthèse dans un monumental recueil de ces textes ${ }^{2}$ - qui a permis une telle découverte ; il l'a rendue publique au cours d'une série de conférences faites au Japon durant l'année 2003. Nous avons assisté à l'une d'elles qui s'est tenue au Tōdaiji de Nara, le 20 décembre 2003, dans le cadre d'un « Symposium sur le Buddha géant de Nara » (« The Great Buddha Symposium ») ${ }^{3}$.

1. Ce religieux a longtemps passé pour être un moine coréen venu au Japon. On pense maintenant qu'il est plutôt un moine japonais qui est allé étudier en Corée et en est revenu. Son appellation coréenne est donnée sous toute réserve ; elle est parfois usitée comme telle aujourd'hui encore dans le monde scientifique coréen.

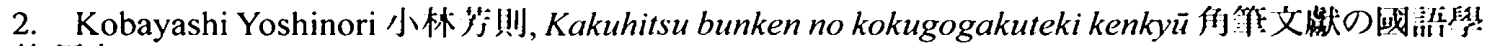
的研究 [Recherches sur les manuscrits avec des caractères tracés au poinçon pour l'étude de la langue japonaise], deux volumes, volume $1:$ Kenkyūhen 研究篇 [Recherches], volume 2 : Eiin shiryōhen 路

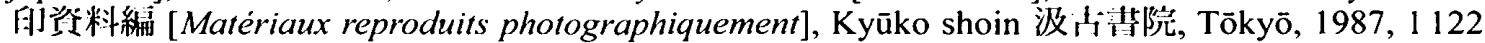
+254 pages.

3. Kobayashi Yoshinori, « Nara jidai no kakuhitsu kunten kara mita Kegonkyō no kōsetsu » [ Les cours sur le Sûtra de l'Ornementation fleurie du point de vue des signes portés au poinçon à l'époque de Nara »], dans The Great Buddha Symposium ronshū, II, Ronshū Tōdaiji sôken zengo, Tōdaiji, 2004, p. 56-73. 
Les documents et manuscrits tracés au poinçon sont connus de la Chine depuis l'Antiquité, au moins depuis l'époque des Han - on a des exemplaires de tablettes en bois, ou des textes de Dunhuang qui en témoignent. Au Japon, on a fait leur découverte depuis quatre décennies environ et, à ce jour, on a recensé quelque trois mille deux cents documents et manuscrits comportant de tels signes, et il ne fait pas de doute que l'usage du poinçon au Japon a son origine sur le continent asiatique. Mais au cours de l'année 2003, l'attention du professeur Kobayashi a été attirée par la découverte de documents et de manuscrits de même type en Corée, datant de la fin du VII ${ }^{\mathrm{e}}$ siècle jusqu'au XIX ${ }^{\mathrm{e}}$ siècle, à partir d'une enquête faite sur la première édition imprimée du Canon bouddhique en Corée, datant du $\mathrm{XI}^{\mathrm{e}}$ siècle. La découverte de signes de lecture de couleur marron du coréen ancien sur une copie japonaise, du début du $\mathrm{Ix}^{\mathrm{e}}$ siècle et appartenant anciennement à $\mathrm{M}$. Satō Tatsujirō 佐藤辰二郎, de l'ouvrage du moine de Silla du Hŭanglyongsa 皇龍寺, Phyowŏn 表員 (?-?), le Hŭa.ŏm mun.ŭi yo.kyŏl 華嚴文義要決, Résolution essentielle du sens du texte de l'Avatamsakasūtra, a attiré l'attention de spécialistes qui y ont vu une attestation de la langue coréenne du viII ${ }^{\mathrm{e}}$ siècle, qui pouvait en même temps avoir influé sur la formation des signes de lecture et d'écriture japonais servant à lire les textes chinois (les wokototen ヨコト點). La découverte d'un ouvrage du moine coréen Wŏnhyo 元曉 (617-686), comportant le sceau de la bibliothèque de l'impératrice Kōmyō, le Traité déterminant le critère de vérité du raisonnement (Panpi lianglon 判比量論), composé en 671 et conservé à l'université Ōtani (Kyoto), a donné une preuve supplémentaire de l'existence de signes et symboles d'écriture du Silla tracés au poinçon, à dater de la première moitié du viII ${ }^{\mathrm{e}}$ siècle. Ces découvertes ont été ensuite mises en relation avec celle de signes de lecture tracés au poinçon parmi les copies de textes bouddhiques de l'époque de Nara.

Une conséquence de ce faisceau de découvertes est que l'on est contraint de faire reculer les débuts des signes de lecture des textes chinois au Japon, du début de l'époque de Heian jusqu'à l'époque antérieure de Nara au moins. Une autre en est que ces signes de lecture des textes chinois au Japon sont à mettre en rapport étroit avec les signes de lecture coréens du Silla, et qu'ils font partie d'un même ensemble attestant d'usages culturels et linguistiques communs qui sont à envisager de manière globale dans cette région géographique.

Les activités de lecture du Collationnement par Shinjō sont historiquement bien attestées, et plusieurs volumes de copies de l'ouvrage, datant de l'époque de Nara et du début du IX ${ }^{\mathrm{e}}$ siècle, nous sont parvenus : les volumes II, V, VIII, IX, XIII, en totalité ou en partie. Il apparaît que des signes de lecture au poinçon - parfois mêlés à des signes d'inversion à la coréenne au pinceau blanc, vermillon, ou à l'encre noire datant sûrement de l'époque de Nara, sont attestés sur le volume V (antérieur à 783), le volume XIII (antérieur à 758, et 794-796), les volumes II et IX (antérieurs au $\mathrm{IX}^{\mathrm{e}}$ siècle ou à 787 ?). Leur examen permet de conclure que les signes portés sur ces textes avant la date de 783 servaient à donner l'ordre de lecture des caractères, tandis que ceux qui sont postérieurs à cette date correspondent surtout à des signes de lecture des caractères, ce qui correspond à un nouveau type dominant à partir de l'époque de Heian.

L'examen des signes portés au poinçon sur le texte même du Sütra de l'Ornementation fleurie (Huayanjing) fait apparaître des signes retraçant les lignes mélodiques imprimées 
sur les textes à psalmodier, les tons de la prononciation chinoise (fushihakase 節博士, littéralement « docteur en tons » ${ }^{4}$ ), des signes de lecture de la prononciation en sinojaponais ou en japonais, des incises de commentaires, des découpages en sections, etc. ${ }^{5}$.

Parmi les autres textes bouddhiques portant ces signes au poinçon, mentionnons le Vinaya des Mülasarvāstivādin 根本一切有部昆奈耶藥事 (volume II, T. XXIV, $\mathrm{n}^{\circ}$ 1448), en date de 768, le Traité des terres des maîtres de Yoga (Yogacārabhūmisāstra) 瑜伽師地論 (T. XXX, n 1579) (volumes LXX, LXXI, LXXIV, LXXVII, LIIIIV, XC, XCIX, C), en date de 744, le volume LVII du même traité, copié durant l'époque de Nara (conservé au Ishiyamadera), le Traité du joyau dans la main du Grand Véhicule (Hastamani) 大乘掌珍論 de Bhavya-Bhāvaviveka (volume I, T. XXX, $\mathrm{n}^{\circ} 1578$ ), en date de 772, le Sütra de la descente à Ceylan (Lañkāvatārasūtra) 楞伽經 (volume IV, t. XVI, n ${ }^{\circ}$ 670-672), en date de 768, et le Sütra de la contemplation sur l'océan du samādhi du Buddha (Guanfosanmeihaijing 觀佛三昧海經, Buddhānusmrtisam ädhisāgarasūtra*) (volume I, T. XV, n 643), en date de 768.

L'usage du poinçon fait apparaître que, lors de la lecture et de l'explication des textes, notamment des sütra, on lisait ceux-ci tout d'abord en sino-japonais, puis on en proposait une lecture en japonais. Tel est le cas du Sütra du Lotus et du Sütra des rois excellentissimes, qui étaient lus de cette manière en date du $15 \mathrm{du} 11^{\mathrm{e}}$ mois de 742 [Tenpyō 14]. On traçait à cette occasion les lignes mélodiques des chants psalmodiés (bonbai) - le fait est attesté en 783, pratique qu'on retrouve à Silla en 755 -, et l'on notait des signes de lecture en japonais, en caractères du type Man.yō ou déjà en caractères simplifiés. Ce dernier type de signes fait partie des nouvelles découvertes qui ouvrent des perspectives considérables pour l'étude de la langue japonaise de l'époque de Nara. Si l'on ne voit pour l'époque de Nara aucun exemple de wokototen, on trouve en revanche des cas de transcription du syllabaire coréen de la langue de Silla et des tons en chinois, dans le traité de Wŏnhyo déjà mentionné ${ }^{6}$.

L'examen des signes portés au poinçon sur les documents et les manuscrits de l'époque de Nara, dont les plus anciens remontent aux années 740, fait en résumé apparaître deux usages principaux de ces « signes invisibles » à l'œil nu : le tracé des lignes mélodiques des psalmodies ; les transcriptions et signes de lecture relatifs aux caractères chinois. On notera les utilisations principales suivantes, telles qu'elles ont été dégagées par le professeur Kobayashi, à propos de textes comportant déjà des signes de lecture et de transcription au pinceau en vermillon :

4. L'étymologie du mot hakase est controversée ; il pourrait signifier « mesure mélodique des tons ».

5. L'examen a porté sur le volume XLI de la version en quatre-vingts volumes, copié en 768 , et sur les volumes XII et XX copiés au vIII' siècle.

6. Kobayashi Yoshinori, «Ōtani daigakuzō shinshutsu kakuhitsu bunken ni tsuite» 大谷大學藏 新忺侮筆文献について, 《Les manuscrits au poinçon nouvellement découverts à la Bibliothèque de l'Université Ōtani », Ōtani gakuhō 大谷學報, volume LXXXII-2, mars 2003. Concernant les rapports entre ces signes tracés au Japon et ceux tracés en Corée, voir Kobayashi, « Kankoku ni okeru kakuhitsu bunken $n^{\circ}$ hakken to sono igi - Nihon kokunten tono kankei - 》韓利における角符文献の発見 とその意義 - 日本竍訓点との将係 - [《Découverte de manuscrits tracés au poinçon en Corée et leur signification - en rapport avec les signes de lecture anciens au Japon »], Chōsen gakuhō 朝鮮學報, $n^{\circ} 182,2002$. 
1 - la mention de corrections de caractères : par exemple 今 à lire 令.

2 - la mention de corrections dans l'ordre de lecture des caractères, par des signes d'inversion: par exemple 初中 à lire dans l'ordre 中初.

3 - la mention de lecture de caractères en sino-japonais (on 音).

4 - des indications de lecture de caractères en japonais ( $k$ un 留, à lire to $s u$ とす.

5 - le tracé des lignes mélodiques et des modulations qui s'étalent sur plusieurs lignes de texte à psalmodier.

6 - des modulations portant sur une seule ligne de texte à psalmodier. (Voir fig. 1)

7 - des indications de caractères liés, à lire comme des mots composés ou des nombres à séparer clairement d'autres caractères : par exemple 一法門者...合理...六 依法性而行少. (Voir fig. 2)

8 - l'indication des tons en chinois, à l'aide de points en forme de ronds blancs $。$, portés sur les angles des caractères chinois.

9 - l'indication par soulignement marquant l'importance d'un passage : par exemple 所以然者此觀.
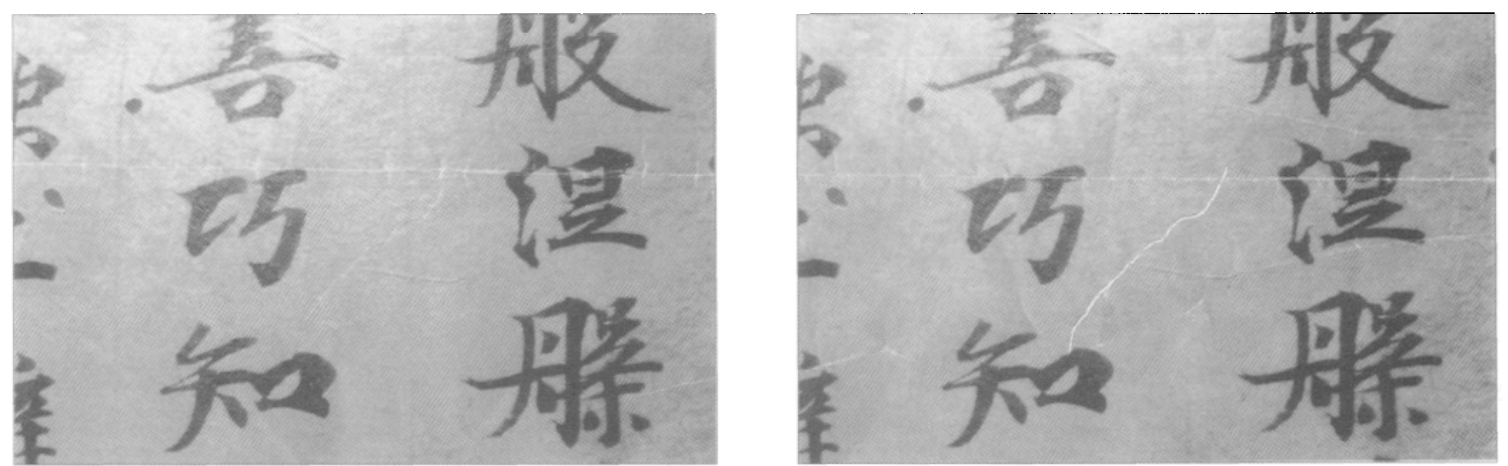

Fig. 1

Tracé d'une ligne mélodique portant sur une colonne de caractères du Sütra de l'Ornementation fleurie (Huayanjing), volume XLI, copié en 768.
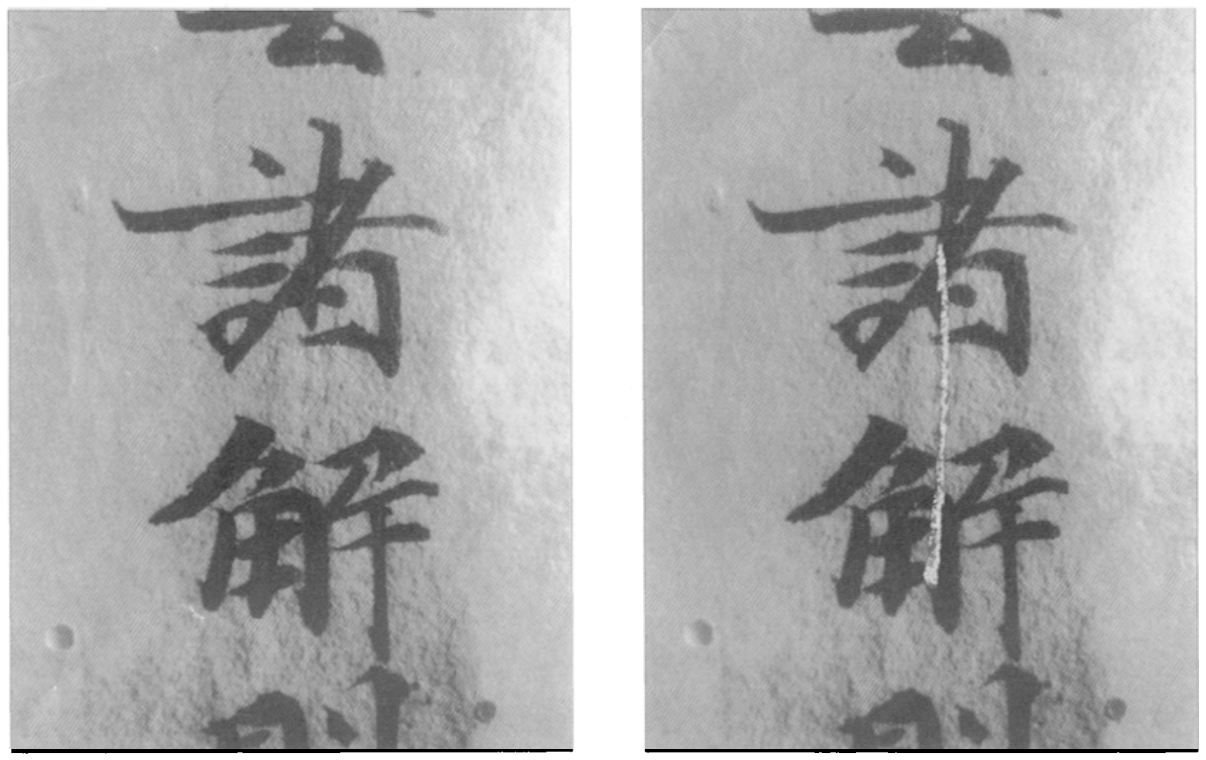

Fig. 2

Liaison de caractères dans le Sütra de l'Ornementation fleurie (Huayanjing), volume XLI, copié en 768. 
Un exemple tiré du volume XLI du Sütra de l'Ornementation fleurie, copié en 768, porte la particule $y i$, transcrite 仪, correspondant apparemment à la particule japonaise prononcée ga. D'après le professeur Kobayashi, c'est selon toute vraisemblance un Coréen qui en est l'auteur. Nous indiquons les signes au poinçon entre crochets dans ce passage que nous pouvons lire : 莊嚴佛子菩薩摩訶薩[仪住[世り]此三昧[尔]或見佛 耳微妙光色[阿畄口]或見佛身...Shōgon busshi bosatsu makasatsu [yi = ga] jū [seri]. kono sanmai [ni], arui wa butsuji no mimyō kōshiki [aru...] wo mi, arui wa busshin wo mi. Nous le reproduisons partiellement ici (voir fig. 3 ci-dessous). Une question se pose : ce $y i$ serait-il la particule coréenne qui correspond au ga japonais? Dans ce cas, comment expliquer son existence dans un document japonais ? Ce $y i$ serait-il le ga que des moines coréens auraient eu tendance à prononcer yi, étant entendu que les auditeurs japonais auraient été en mesure d'en saisir le sens ? Ou bien correspondrait-il à une prononciation effective du ga que nous connaissons et qui, à époque ancienne, ou bien seulement à Nara, se prononçait $y i$ ou d'une façon proche de $y i$ ?

Il importe de signaler à ce propos que de nombreux textes appartenant à la tradition de Nara, les ouvrages de l'école Hossō en particulier qui était, rappelons-le, sise au Kōfukuji, portent trace d'une particule enclitique $i$ qui correspond de façon manifeste à ga en tant que marque du sujet grammatical. Signalons-en quelques exemples notoires.

L'édition japonaise du Hōryūji de la somme de l'école Hossō, la Vijñaptimātratāsiddhi, le Traité qui démontre que tout n'est que notification cognitive, qui collationne des lectures s'étalant de l'époque de Nara jusqu'à celle d'Edo, donne des exemples nombreux de cette lecture :

« Le vijñāna intérieur se développe pareil à des objets extérieurs » arui wa mata, naishiki i tenjite gekyō ni niru 或，復夕内識，似，外界二?.

« La deuxième théorie selon laquelle le moi (âtman) est à part des ensembles (skandha), ne s'érige pas en raison du fait que ce moi n'est ni agent ni récipiendaire de l'action. " naka no iun no ga mo kotowari i mata shikarazu. kokū no gotoku sashi jusuru koto nakaru beki ga yue ni 中ノ離總ノ我乇理,亦不久然，應切如,虚空，無 カル作シ受スルコト故 8 .
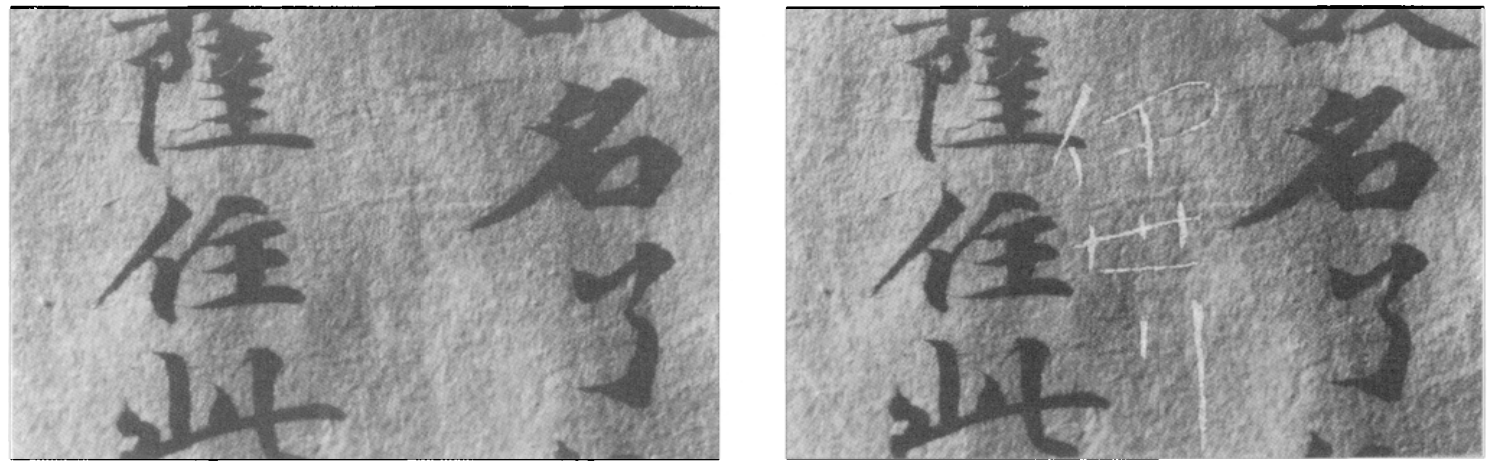

Fig. 3. Signes de lecture de caractères du Sütra de l'Ornementation fleurie, copié en 768.

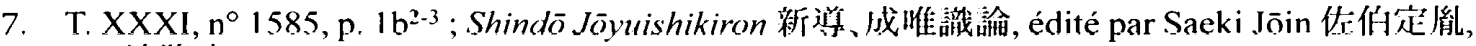
Hōryūji 汒隆杵, Nara, p. 3. Voir traduction de Louis de La Vallée Poussin, p. 9.

8. Ibidem, p. $1 \mathrm{c}^{15-16}$; Shindō Jōyuishikiron, p. 6. Voir traduction de Louis de La Vallée Poussin, p. 14. 
Les moines savants spécialistes de cette doctrine de l'époque de Heian et du début de celle de Kamakura ont compilé un recueil des commentaires de leur école, Jōyuikiron dōgakushō 成唯識論|问学鈔, Recueil de commentaires des condisciples de l'école sur la Vijñaptimātratāsiddhi :

«Cette phase [d'actualisation] parfume les germes et les germes suivent la phase d'actualisation » kono sō ii shū wo kunji, shū [wa/ii ?] gensō ni shitagau 此相你熏 種。種随現相 9 . 疑 10 .

«Ce principe ne fait aucun doute » kotowari ii aru koto utagai nashi 有理々無

Dans un autre ouvrage appartenant à la tradition de Nara, Le Sens des choses selon l'Abhidharmakośa, Abidatsumakusharon hōgi 阿毘達磨俱舍論法義, du moine de l'école du Néo-Shingon, Kaidō 快道 (1751-1810) qui a vécu à Edo, on retrouve ce genre de lecture, ce qui atteste sa persistance dans l'espace comme dans le temps :

« La pensée est susceptible de comporter des résidus psychiques inconscients. Et en comporter c'est ce qu'on appelle être pourvu de résidus psychiques inconscients » kokoro ii yoku zuimin ari, zuimin no aru koto wo yūzuimin to nazuku 心イ能有随 眠。隨眠之有名有隨眠 11 .

Des questions restent pendantes. Il reste à savoir si ce $i$ est le vestige d'une influence du coréen sur le japonais, qui subsisterait à Nara, ou s'il est dès le départ la marque d'une particularité (dialectale ?) de la région de Nara, relevant du seul japonais. Ne peut-on espérer trouver réponse à ces questions grâce à des recherches plus fines effectuées dans cette direction?

Doit-on songer à des faux ou à des adjonctions tardives de signes tracés au poinçon « invisibles », donc à caractère frauduleux ? Presque personne n'avait accès à la bibliothèque du Shōsōin, ce qui réduit à presque rien le nombre d'utilisateurs potentiels de ces textes ; il faudrait expliquer en quelles circonstances on aurait fait usage de copies du Canon commandé par l'impératrice Kōmyō, par exemple lors de cérémonies, et pourquoi un scribe clerc ou laïc aurait tracé des signes « invisibles » à une époque ultérieure à celle de Nara, où l'on avait coutume, s'agissant de sūtra bouddhiques, de tracer de tels signes en couleur.

Cette moisson de documents, acquise chèrement, si l'on songe aux difficultés d'accès des bibliothèques japonaises, surtout celles qui sont liées à la famille impériale ou aux clans shôgounaux, et encore très provisoire, laisse présager d'autres découvertes nouvelles concernant l'histoire de la langue japonaise, le rôle des Coréens dans l'élaboration du syllabaire japonais ainsi que du bouddhisme dans l'histoire ancienne de la civilisation japonaise. Elle nous contraint, dans l'immédiat et en attendant plus ample information, à reculer dans le temps l'invention du syllabaire japonais, qui semble être le résultat de pratiques religieuses et liturgiques dans lesquelles les moines du royaume coréen de Silla ont sans aucun doute joué un rôle de premier plan.

9. T. LXVI, $\mathrm{n}^{\circ} 2263$, p. $93 \mathrm{~b}^{28}$. Ici, la particule est redoublée : ii. Doit-on la lire $y i$ ?

10. Ibidem, p. $8 \mathrm{~b}^{7}$. Ici aussi la particule est redoublée.

11. T. LXIV, $\mathrm{n}^{\circ} 2251$, p. $288 \mathrm{~b}^{1}$. Là aussi la particule est redoublée. 